\title{
Preliminary word-association norms for institutionalized adolescent retardates'
}

\author{
Irma R. Gerjuoy, JOHNSTONE TRAINING AND RESEARCH CENTER, BORDENTOWN, N. J. \\ Herbert Gerjuoy, EDUCATIONAL TESTING SERVCE
}

\begin{abstract}
Abstraet
One hundred institutionalized adolescent educable retardates gave oral free-association responses to the 100 words of the Kent-Rosanoff list presented in an order that was expected to minimize antonym response set. Their responses were tabulated to provide preliminary word-association norms for this subject population.
\end{abstract}

\section{Problem}

Word associations have played an important role in the investigation of verbal behavior. Extensive normative data have been collected from college students (e. g., Russell \& Jenkins, 1954) and more recently from school children (Palermo \& Jenkins, 1964).

The Palermo \& Jenkins norms have been very useful in the assessment of the verbal skills of normal children; in the assessment of the verbal skills of mentally retarded children and adolescents, such freeassociation norms would probably be of equal importance. The only reported norms for word associations of retarded children are Horan's $(1955,1956)$, whose Ss were mentally retarded children from the public schools of New York City. Institutionalized mentally retarded adults were tested by Silverstein \& McLain (1961, 1964); in their 1961 study, however, only 55 of the 102 Ss were testable.

The purpose of the present study was to determine the feasibility of collecting word associations from institutionalized adolescent retardates and to provide some preliminary norms for such a population.

\section{Subjects}

The Ss were 102 adolescent educable retardates residing at the Johnstone Training and Research Center. Two Ss were eliminated: one for refusal to cooperate and one for a severe speech and hearing handicap. The remaining. $100 \mathrm{Ss}, 35$ girls and 65 boys, had a mean chronological age of $16.05 \mathrm{yr}$. ( $\mathrm{SD}=1.66 \mathrm{yr}$.) and a mean IQ of $63.04(\mathrm{SD}=10.00)$; IQ was determined by the most recently administered individual test, which was the WISC in most cases.

\section{Stimuli}

The stimuli were the 100 words in the Kent-Rosanoff list (Kent \& Rosanoff, 1910). To help control antonymresponse set, the set of 54 words selected by Lansdell ${ }^{2}$ was administered first; they were administered in the order that Wynne et al (1965) found eliminated the antonym-response set in normal adults Ss-Wynne et al refer to this order as C2 (see Table 1). The remaining 46 words were presented in the order in which they appeared in the original Kent-Rosanoff list.

\section{Procedure}

The Ss were tested individually by the same female E. Since most of the Ss have very limited reading ability, the oral presentation method was used. Ss who wanted to read the words themselves were given a blank data sheet to follow, but all responses were recorded by the $\mathrm{E}$. Whenever a S's response could have alternative spellings (e. g., "moose" or "mousse"), the same alternative was always arbitarily recorded; the test was not interrupted to question the S. Although the test was not timed, the Ss were encouraged to respond rapidly.

The instructions were as follows: "I am going to read you some words one at a time. After you hear my word, I want you to say one word, the very first word that comes to your mind. Say your word as quickly as you can after I say mine." Whenever a S gave more than one word, he was reminded to say just one word.

\section{Results}

Since only two of the 102 Ss were not testable, the feasibility of collecting word associations from institutionalized adolescent retardates was clearly indicated. The responses to the 100 Kent-Rosanoff stimulus words were tabulated and are presented in full in Gerjuoy \& Gerjuoy (1964). The stimuli are listed in the order of administration, and following each stimulus is an alphabetical list of the responses to that word and their frequencies. The list of stimuli is also given in the order of administration in Table 1 along with the most popular responses and their frequencies.

Table 1. Stimuli Listed in Order of Administration and the Most Popular Responses and Their Frequencies. The words following the colon are responses, and the numbers in parentheses are the frequencies of responses. 100 subjects were used, and hence 100 responses to each stimulus were obtained.

1 Tobacco: cigarette (42), pipe (19), cigarettes (9), smoke (7), chew (4) 2 Cottage: one (14), house (13), building (7)

3 Scissors: cut (63), paper (10), knife (9), clippers (2)

4 River: water (38), ocean (14), swim (10), lake (9), delaware (6)

5 Whiskey: drink (37), wine (20), beer (15), alcohol (5), liquor (5)

6 Bed: sleep (69), couch (6)

7 Eagle: bird (56), fly (20), animal (2), owl (2)

8 Memory: think (20), mind (16), (no response, 12), remember (11)

9 Religion: catholic (26), church (23), protestant (13), (no response, 13)

10 Stomach: body (15), food (11), eat (10), ache (8)

11 Health: (no response, 8), clean (6), good (6), sick (6),

12 Afraid: scared (67), frightened (4)

13 Green: grass (24), color (19), red (14), yellow (6), blue (5)

14 Deep: hole (17), water (16), down (12), cut (5), high (4), low (4)

15 Child: baby (32), children (19), boy (18), girl (6), kid (4), small (4)

16 Eating: food (32), ate (9), hungry (6), eat (4)

17 Thief: steal (44), robber (12), stealing (9), crook (7) 
18 Smooth: soft (27), rough (11), (no response, 9), hard (5), nice (4) 19 Hungry: eat (24), starved (20), food (14), starving (13)

20 Sickness: sick (15), hospital (8), disease (7)

21 Lamp: light (68), bulb (5), shade (5), lamb (3), table (3)

22 Square: round (22), box (18), block (17), triangle (9), circle (8)

23 Ocean: river (38), water (21), sea (16), lake (8), boat (3)

24 King: Queen(52), man (8), (no response, 7), boss (4)

25 Salt: pepper (48), sugar (14), food (12), eat (4), shaker (3)

26 Needle: thread (41), sew (15), pin (13), sharp (5), medicine (3)

27 Rough: smooth (16), tough (14), hard (8), soft (7)

28 Sweet: sugar (21), candy (17), sour (17), bitter (9)

29 Hammer: nail (52), nails (14), bang (6)

30 Boy: girl (64), child (6), man (6), baby (3), human being (3)

31 Butter: bread (49), knife (6), milk (6), spread (6), eat (5), food (5)

32 High: low (36), good-by (7), hello (6)

33 Dream: sleep (34), nightmare (9), think (7), (no response, 5)

34 Thirsty: water (61), drink (20), dry (5), hungry (4), soda (2)

35 Girl: boy (55), woman (11), child (5), female (3), lady (3), man (3)

36 Chair: sit (45), table (18), seat (8), desk (5), sit down (5)

37 Bitter: sour (20), sweet (15), (no response, 15), taste (7), better (6)

38 Slow: fast (56), walk (9), learn (4), learning (3), walking (3)

39 Fruit: vegetable $(20)$, apple $(18)$, eat $(14)$, orange $(10)$

40 Man: woman (45), boy (14), lady (6), father (5)

41 Stem: tree (20), leaf (19), flower (14), branch (7), stick (7)

42 Sour: bitter (24), sweet (14), milk (10), taste (6), (no response, 5)

43 Head: hair (19), body (12), eyes (7), face (6), shoulder (6)

44 Black: white (26), color (11), crayon (10), blue (6), yellow (6)

45 Swift: (no response, 30), fast (11), kick (8), cheese (4)

46 Soft: hard (28), smooth (14), rough (7), bread (5), bed (4)

47 Hand: arm (16), fingers (15), feet (9), finger (8)

48 Woman: man (40), lady (25), girl (15), mother (3)

49 House: home (35), live (10), cottage (6), building (5)

50 Heavy: light $(40)$, hard (9), weight $(9)$, fat $(6)$, rock (3)

51 Light: heavy (19), bulb (12), dark (10), lamp (8), skinny (4)

52 Long: short (46)

53 White: black (27), paper (9), color (8), light (5), red (5)

54 Hard: soft (38), easy (6), rock (5), heavy (4), wood (4), work (4)

55 Table: chair (44), eat (7), desk (6), chairs (4), food (4)

56 Dark: light (46), night (17), day (5), black (4), midnight (3)

57 Music: song (13), sing (11), singing (8), instrument (7), piano (7)

58 Mountain: hill (22), high (15), rocks (14), climb (13), rock (6)

59 Mutton: (no response, 59), mitten (6), button (5)

60 Comfort: (no response, 20), relax (12), comfortable (11), chair (8)

61 Short: long (34), tall (20), pants (8), small (7), boy (3)

62 Butterfly: fly (21), animal (15), caterpillar (9), bird (7), insect (6)

63 Command: (no response, 18), order (7)

64 Whistle: blow (32), horn (8), bird (7), noise (4), tune (4)

65 Cold: hot (29), warm (19), sick (10), freezing (5), cough (4)

66 Wish: true (10), (no response, 6)

67 Beautiful: pretty (22), ugly (19), nice (17), lovely (6), woman (4)

68 Window: glass $(20)$, pane $(11)$, open $(8)$, frame $(7)$

69 Citizen: (no response, 18), people (15), country (7)

70 Foot: feet $(31)$, leg $(18)$, toe (8), shoe (7), walk (7)

71 Spider: web (33), animal (12), insect (12), bug (7), crawl (3), fly (3)

72 Red: blue (15), color (15), crayon (8), white (7), yellow (7)

73 Sleep: bed (31), awake (8), wake (8), dream (6), tired (6)

74 Anger: mad (39), angry (11), (no response, 9), mean (7), fight (3)

75 Carpet: rug (37), (no response, 10), floor (8), wood (4)

76 Working: hard (22), job (11), playing (11), men (3), school (3)
77 Earth: world (20), ground (13), quake (8), round (7), people (6) 78 Trouble: bad (13), maker (10), fight (9)

79 Soldier: army (38), man (9), boy (8), navy (7), marching (6) 80 Cabbage: food (24), lettuce (18), eat (16), vegetable (15), fruit (5) 81 Yellow: color (17), green (13), crayon (8), red (8)

82 Bread: butter (33), eat (21), food (15), milk (3), soft (3), wheat (3) 83 Justice: peace (22), (no response, 20), law (10), court (5), judge (4) 84 Bible: read (19), book (14), God (12), holy (12)

85 Sheep: lamb (37), animal (21), cow (4), goat (4)

86 Bath: tub (19), wash (19), shower (17), water (17), clean (11) 87 Blue: color (15), sky (11), red (10), white (9), crayon (8), purple (8) 88 Priest: minister (28), father (16), church (12)

89 Stove: cook (39), oven (12), fire (11), hot (10), kitchen (5)

90 City: town (31), country (22), state (11), people (5), New York (4) 91 Doctor: nurse (32), help (8), hospital (7), medicine (6), sick (5) 92 Loud: soft (20), low (11), scream (8), quiet (7), talk (7)

93 Lion: tiger (41), animal (27), cat (5), roar (4)

94 Joy: happy (35), fun (12), boy (5), (no response, 5), glad (4) 95 Baby: boy (23), child (21), mother (7), girl (5), small (5)

96 Moon: sun (37), light (9), earth (8), night (5), shine (5), sky (5) 97 Quiet: noisy (16), loud (15), noise (8), sleep (8)

98 Street: road (32), cars (10), walk (10), car (6), city (5)

99 Cheese: rat (23), eat (21), food (9), mice (6), bread (5)

100 Blossom: flower (57), flowers (13), blue (3), (no response, 3)

\section{References}

GERJUOY, I. R., \& GERJUOY, H. Preliminary word-association norms for institutionalized adolescent retardates. Research Memorandum 64-14. Princeton, N.J.: Educational Testing Service, 1964. (Multilithed report)

HORAN, E. M. Word association frequency tables of mentally retarded children. Unpublished doctoral dissertation, Columbia University, 1955.

HORAN, E. M. Word association frequency tables of mentally retarded children. J. consult. Psychol., 1956, 20, 22.

KENT, G. H., \& ROSANOFF, A. J. A study of association in insanity. Amer. J. Insan., 1910, 67, 37-96, 317-390.

PALERMO, D. S., \& JENKINS, J. J. Word association norms; grade school through college. Minneapolis: Univ. Minnesota Press, 1964.

RUSSELL, W. A., \& JENKINS, J. J. The complete Minnesota norms for responses to 100 words from the Kent-Rosanoff word association test. Technical Report No. 11. Contract N8-ONR-66216, ONR. Minneapolis: Univ. Minnesota, 1954.

SILVFRSTEIN, A. B., \& McLAIN, R. E. Associative processes of the mentally retarded: I. An exploratory study. Amer. J. ment. Defic., 1961, 65, 761-765.

SILVFRSTEIN, A. B., \& MCLAIN, R. E. Associative processes of the mentally retarded: II. Effects of selected background variables. Amer. J. ment. Defic., 1964, 69, 440-445.

WYNNE, R. D., GERJUOY, H., \& SCHIFFMAN, H. Association test autonym-response set. J. verb. Learn. verb. Behav., 1965, 4, in press.

\section{Notes}

1. We thank Sydell T. Carlton, Sandra R. Cohen, and David Rosenhan for editorial advice and Arthur M. Adlerstein and Norman Wexler for help in tabulating the results.

2. Herbert Lansdell, unpublished word-association test, National Institutes of Neurological Diseases and Blindness, NIH, 1959. 